\title{
Análise do Comportamento e Saúde: Levantamento e Análise de Artigos em Bases de Acesso Livre
}

\author{
Taís da Costa Calheiros ${ }^{1}$ \\ Programa de Pós-Graduação em Análise do Comportamento da Universidade Estadual \\ de Londrina, Londrina, PR, Brasil \\ Mariana Amaral \\ Programa de Pós-Graduação em Psicologia Experimental: Análise do Comportamento \\ da Pontifícia Universidade Católica de São Paulo, São Paulo, SP, Brasil \\ Centro Universitário Filadélfia, Londrina, PR, Brasil \\ Márcia Cristina Caserta Gon \\ Departamento de Psicologia Geral e Análise do Comportamento da Universidade Estadual \\ de Londrina, Londrina, PR, Brasil \\ Áderson Luiz Costa Júnior \\ Departamento de Psicologia Clínica da Universidade de Brasília, Brasília, DF, Brasil
}

\begin{abstract}
Resumo
Diante das críticas à falta de sistematização da prática psicológica na área da saúde, objetivou-se apresentar uma revisão sistemática em bases de dados on-line de acesso livre e analisar artigos científicos analítico-comportamentais, publicados entre 2005 e 2014, na área da Psicologia da Saúde. Foram feitos dois procedimentos na Biblioteca Virtual em Saúde (BVS) - Psicologia Brasil, especificamente no Index Psi Periódicos Técnico-Científicos, na Scientific Electronic Library On-line e na Literatura Latino-Americana e do Caribe em Ciências da Saúde. Na Coleta 1, foi utilizada a estratégia de busca "análise do comportamento" $A N D$ saúde $O R$ "medicina do comportamento" OR "psicologia médica", cujos termos deveriam constar como "palavras" ou "em todos os índices". Na Coleta 2, utilizou-se "psicologia" AND outros termos referentes a 45 especialidades da Medicina, presentes no "resumo". A Ficha Cadastral dos Artigos Científicos orientou a análise quanti-qualitativa em função de sete itens: referência, ano de publicação, tipo de estudo, objetivos, participantes, procedimentos e conclusões. Os resultados sugeriram a aplicabilidade da Análise do Comportamento à área da saúde, ainda que o número de artigos recuperados tenha sido restrito devido a entraves conceituais e metodológicos. Verificou-se a necessidade de aproximação dos critérios de análise desses materiais pelos profissionais da Ciência da Informação e da Análise do Comportamento.
\end{abstract}

Palavras-chave: Psicologia da Saúde, Medicina do Comportamento, Literatura de Revisão.

\footnotetext{
Endereço para correspondência: Universidade Estadual de Londrina, Departamento de Psicologia Geral e Análise do Comportamento, Rodovia Celso Garcia Cid (PR 445), Km 380, CEP 86057-970, Caixa Postal 10011, Londrina, PR, Brasil. Fone: +55 433371-4203 /+55 439831-1111. E-mail: calheirostais@gmail.com. Financiamento: Coordenação de Aperfeiçoamento de Pessoal de Nível Superior (CAPES) e Fundação Araucária.
} 


\title{
Behavior Analysis and Health: Survey and Analysis of Articles in Open Access Databases
}

\begin{abstract}
In the face of criticism on the lack of a systematization of psychological practice in health, was aimed to present a systematic review in open access on-line databases and analyze the scientific articles analytical behavior, published between 2005 and 2014, in Health Psychology. Two procedures were made in VHL Psychology Brazil, specifically in the Index Psi Technical-Scientific Journals, the Scientific Electronic Library On-line and the Latin American and Caribbean Health Sciences Literature. In Collection 1, was used the search strategy "behavior analysis" AND health OR "behavioral medicine" OR "medical psychology", which terms should be included as "words" or "in all indexes". In Collection 2, was used "psychology" AND other terms referring to 45 specialties of Medicine, present in the "abstract". The Registration Form of Scientific Articles guided the quanti-qualitative analysis on the basis of seven items: reference, year of publication, type of study, objectives, participants, procedures and conclusions. The results suggested the applicability of Behavior Analysis to healthcare, although the number of recovered items has been restricted due to conceptual and methodological obstacles. A need was seen to approach the analysis criteria of these materials by Information Science and Behavior Analysis professionals.
\end{abstract}

Keywords: Health Psychology, Behavioral Medicine, Review Literature.

\section{Análisis del Comportamiento y Salud: Levantamiento y Análisis de Artículos en Bases de Datos de Acceso Abierto}

\section{Resumen}

Este estudio tuvo como objetivo presentar una revisión sistemática en las bases de datos on-line de acceso abierto y analizar artículos científicos analítico-comportamentales, publicados entre 2005 y 2014, en la Psicología de la Salud. Dos procedimientos fueron realizados en la Biblioteca Virtual en Saúde (BVS) Psicología Brasil, específicamente en el Index Psi Revistas Técnico-Científicas, en la Scientific Electronic Library On-line y en la Literatura Latinoamericana y del Caribe en Ciencias de la Salud. En la Recolecta 1, fue utilizada la estrategia de busca "análisis del comportamiento" $A N D$ salud $O R$ "medicina del comportamiento" $O R$ "psicología médica", cuyos términos deberían constar como "palabras" o "en todos los índices". En la Recolecta 2, se utilizó "psicología" AND otros términos referentes a 45 especialidades de la Medicina, presentes en los "resúmenes". El análisis cuanti-cualitativo constó de siete ítems: referencia, año de publicación, tipo de estudio, objetivo, participante, procedimientos y conclusiones. Los resultados sugirieron la aplicabilidad del Análisis del Comportamiento en el área de la salud, aunque el número de artículos recuperados haya sido restricto debido a los obstáculos metodológicos y conceptuales. Se verificó a necesidad de aproximación de los criterios de análisis de esos materiales por profesionales de la Ciencia de la Información y del Análisis del Comportamiento.

Palabras clave: Psicología de la Salud, Medicina del Comportamiento, Literatura de Revisión.

A Psicologia da Saúde, um campo de contribuições científicas e profissionais específico da Psicologia, tem seus objetivos centralizados na promoção e manutenção da saúde, prevenção e tratamento de doenças e de disfunções em pessoas habitualmente saudáveis (Matarazzo, 1980).
Essa especialidade apresenta natureza educacional, multiprofissional e interdisciplinar, direcionada à identificação de etiologias e diagnósticos relacionados à saúde, doenças e disfunções, bem como à análise do aprimoramento e regulamentação do sistema de atenção à saúde e da for- 
mação de políticas públicas. Tais intervenções devem gerar conhecimentos que solucionem problemas do sistema de saúde, incluindo tanto a relação entre comportamento e saúde, quanto o aprimoramento de recursos humanos e de instituições de saúde (Costa, 1997).

Por vezes, utilizam-se os termos Psicologia Clínica e da Saúde, Medicina Psicossomática, Psicologia Hospitalar (Kerbauy, 2002) e, erroneamente, Psicologia Clínica (Castro \& Bornholdt, 2004) para referir-se à mesma subárea, o que indica uma recorrente confusão de terminologias, com denominação problemática e fundamentada em referenciais teóricos distintos (Kerbauy, 2002). De qualquer maneira, o psicólogo da saúde deve ser entendido como o profissional que lida com problemas relacionados ao continuum saúde/doença, independentemente do espaço no qual esteja inserido (Miyazaki \& Amaral, 1995), tendo como funções primordiais auxiliar na compreensão das relações existentes entre comportamento e saúde (Costa, 1997) e estimular o estabelecimento de relações cooperativas entre o paciente, a família e a equipe de saúde (Conselho Federal de Psicologia, 2006).

Diversas abordagens psicológicas fazem parte do campo de ensino, pesquisa e intervenção denominado Psicologia da Saúde. Desde o início do desenvolvimento da área, a Análise do Comportamento tem demonstrado empiricamente contribuições importantes para a compreensão dos fatores relacionados ao desenvolvimento de doenças, à promoção e manutenção de comportamentos de saúde e à modificação de comportamentos de risco em diferentes contextos (Jenkins, 2003/2007). Nessa abordagem, o comportamento é visto como fator determinante no processo saúde-doença e, assim, intervenções comportamentais envolvendo indivíduos e grupos podem ser planejadas com objetivos de promoção, prevenção e tratamento (Straub, 2005). Essas intervenções são direcionadas a eventos relacionados à ocorrência do comportamento, sejam eles classes de estímulos antecedentes ou consequentes.

No Brasil, as intervenções psicológicas na área da saúde tornaram-se gradativamente pauta- das em conhecimentos científicos, sendo imprescindível uma formação específica para esse exercício profissional (Castro \& Bornholdt, 2004). Nessa perspectiva, os psicólogos, em especial os analistas do comportamento, necessitam de treinamento a fim de refinar habilidades complexas que lhes permitam atuar eficazmente na prestação de serviços e/ou em atividades de pesquisa, sendo resguardadas as devidas distinções entre a pesquisa aplicada e os serviços prestados diretamente à população na área da saúde (Johnston, 1996).

Parte desse conhecimento advém do contato com a produção científica da área, a qual fornece o embasamento teórico e o modelo prático que devem sustentar a atuação do profissional. Uma vez que houve uma reestruturação marcante nos meios de publicação com o advento da Internet, essa se caracteriza, atualmente, como uma das mais acessíveis fontes de informação técnico-científica que pode respaldar o trabalho na Psicologia (Packer, 2005).

Essa alteração promoveu impactos em diferentes instâncias do ciber-espaço, as quais estão atreladas também à dimensão política dessa divulgação: o conhecimento passa a ser preconizado como bem público e os movimentos de open access estão se fortalecendo (Packer, 2005). Nesse sentido, a Biblioteca Virtual em Saúde Psicologia (BVS - Psi Brasil) é um exemplo de projeto de controle de publicação da literatura nacional em Psicologia, ${ }^{2}$ visando à recuperação eficiente e rápida da informação técnico-científica, com um acesso universal e equitativo. Esse tipo de iniciativa é vantajoso, pois uma maior acessibilidade a bases de dados como essa torna possível a aproximação de todos os profissionais do conhecimento científico que deve sustentar suas atuações. Essa iniciativa incluiria a maior parte dos psicólogos, já que esses se encontram afastados das universidades (e.g., $87,8 \%$ no Paraná), de acordo com levantamento feito pelo Conselho Regional de Psicologia $8^{\text {a }}$ Região (2010). Por não estarem inseridos em

2 Recuperado de http://www.bvs-psi.org.br/php/ level.php?lang $=$ pt\&component $=19 \&$ item $=30$ 
meios acadêmicos, esses profissionais, de modo geral, não têm acesso a bases de dados de acesso restrito, cujas publicações são mais numerosas e tendem a estudar fatores que vão além da doença (Witter, 2008). Muitas dessas publicações estão disponíveis em língua estrangeira, o que também pode influenciar na consulta feita por vários desses profissionais.

Diante das peculiaridades verificadas na divulgação científica atual, das críticas à ausência de práticas psicológicas sistematizadas nos ambientes médicos (Costa et al., 2012; Gorayeb \& Guerrelhas, 2003), da noção dos desafios históricos enfrentados pelos psicólogos em relação à formação profissional, inserção e correspondência às expectativas do mercado de trabalho da saúde (Glueckauf, 1999; King, 2006; Matarazzo, 1982; McNamara, 1981) e do reconhecimento de que as intervenções comportamentais geram a condição de saúde por meio de tratamentos com uma relação custo-benefício vantajosa e efetiva (Kaplan, 1990), entende-se como relevante investigar a produção científica brasileira de analistas do comportamento na Psicologia da Saúde disponível em bases de dados de acesso livre. Pretende-se apresentar e discutir características dos estudos recentes com embasamento analítico-comportamental que estão ao alcance de todos os profissionais da Psicologia, permitindo a aproximação entre a área médica e esse sistema psicológico, além de favorecer a atuação sob uma perspectiva unificada baseada em estudos sobre comportamento e saúde (Pomerleau, 1979; Reid, 1991; Smith, Kendall, \& Keefe, 2002), com práticas mais efetivas às demandas de atendimento.

A presente pesquisa, portanto, teve como objetivos: (a) apresentar o processo de execução de uma revisão sistemática a partir de bases de dados on-line de acesso livre e (b) analisar os artigos científicos analítico-comportamentais referentes à Psicologia da Saúde publicados no período de 2005 a 2014. Especificamente, neste último, dedicou-se a: localizar os artigos científicos, quantificá-los por ano de publicação e analisá-los quanto ao tipo de estudo, objetivos, procedimentos, participantes, no caso de pesquisas aplicadas ou experimentais, e conclusões.

\section{Método}

O presente trabalho consiste em uma revisão sistemática, a qual se caracteriza pelo processo de reunião e avaliação de várias pesquisas de maneira crítica e sintética (Costa \& Zoltowski, 2014). Essa síntese rigorosa da literatura fazse necessária em razão do fluxo contínuo de informações que são publicadas e interferem no processo de tomada de decisão dos profissionais da área da saúde, bem como do tempo limitado desses, o que tem gerado a necessidade de desenvolvimento de estudos concisos (Galvão, Sawada, \& Trevizan, 2004).

\section{Instrumento}

A Ficha Cadastral dos Artigos Científicos estabelece os critérios para a coleta, tabulação e análise dos dados, sendo composta por sete itens, dos quais seis correspondem às variáveis de estudo para a análise quantitativa e qualitativa desta revisão sistemática, a saber: Referência, Ano de Publicação, Tipo de Estudo, Objetivos, Participantes, Procedimentos e Conclusões.

\section{Procedimento}

Seleção das Bases de Dados. Foi efetuado acesso remoto às fontes citadas no Comunicado n. 001/2013 do Ministério da Educação (2013), referente à atualização dos critérios do WebQualis da área da Psicologia. Esse documento foi obtido por meio da consulta aos critérios Qualis por área, selecionando a Psicologia como área de avaliação e o período correspondente ao ano de 2011, em virtude do documento relativo à classificação do ano de 2012 ainda não estar disponível no momento da consulta. Todas as fontes de dados listadas foram consultadas para a verificação de acesso livre.

A BVS é um espaço virtual coordenado e implantado pelo Centro Latino Americano e do Caribe de Informação em Ciências da Saúde (BIREME) sob a liderança da Organização Pan-Americana da Saúde (OPAS) / Organização Mundial da Saúde (OMS). Objetiva integrar a informação técnico-científica produzida nas $\mathrm{Ci}$ ências da Saúde e desenvolver um controle da 
publicação científica brasileira em Psicologia (Packer, 2005). Nesse âmbito, hipotetizou-se que a comunidade acadêmica e profissional da área consulte, corriqueiramente, trabalhos concisos e de qualidade, como os indexados na BVS, o que determinou a seleção dessa fonte de dados.

No entanto, em função da BVS-Psi Brasil incorporar várias outras bases, foram selecionadas três bases de dados para a realização da coleta de dados dessa revisão, sendo estas: Index Psi Periódicos Técnico-Científicos, Scientific Eletronic Library On-line (SciELO) e Literatura Latino-Americana e do Caribe em Ciências da Saúde (LILACS). Cada uma delas consiste em um dos três tipos de bases de dados existentes na BVS-Psi Brasil: a primeira é uma base de artigos científicos de abrangência nacional que contém referências com resumos, a segunda é uma base de dados eletrônica com textos completos e a terceira é um índice de publicações das Ciências da Saúde, cujos registros são indexados no Google.

O idioma escolhido foi o Português, hipotetizando a consulta feita por profissionais e discentes da área que não tenham o domínio da leitura em língua estrangeira. Entende-se que, embora esse critério não esteja, necessariamente, relacionado à competência profissional ou acadêmica desses indivíduos, nem sequer tenha correlação com a qualidade das intervenções desenvolvidas, sabe-se que o contato com referências bibliográficas internacionais está restrito a instituições e populações específicas, bem como ocorre com a aprendizagem de outros idiomas. Portanto, esses fatores podem restringir a literatura consultada e não caracterizar o que vem orientando a formação e a atuação dos acadêmicos e psicólogos.

Seleção dos Descritores. A BVS-Psi Brasil dispõe o vocabulário controlado Descritores em Ciências da Saúde (DeCS), o qual foi utilizado como padrão para a seleção dos descritores empregados nessa pesquisa. Pellizzon (2004, p. 153) afirma que o "uso de um vocabulário estruturado permite ao pesquisador recuperar a informação com o termo exato utilizado para descrever o conteúdo daquele documento científico". Portanto, os descritores são essenciais para o processamento da revisão, atuando como um filtro entre a terminologia de uma área e a linguagem do autor.

Fez-se necessária, portanto, a construção de uma relação de termos que auxiliasse a circunscrever o âmbito da literatura a ser estudada (Luna, 2007). Foi elaborada uma lista de palavras-chave utilizadas em trabalhos científicos de autores de referência na Análise do Comportamento ${ }^{3}$ que produzem sobre temáticas relativas à Psicologia da Saúde, sem que essas fossem, necessariamente, parte do conjunto dos descritores exatos do DeCS.

Inicialmente, a inserção das palavras-chave "análise funcional" e "terapia comportamental" na BVS-Psi Brasil foi feita entre aspas e isoladamente, de forma a recuperar trabalhos que fizessem uso desses termos. A partir de então, a primeira autora registrava os Descritores DeCS e Descritores Psi Português dos trabalhos pertencentes à interface de interesse. Na sequência, todos esses termos foram inseridos no DeCS, a partir dos seguintes critérios: o idioma selecionado foi o "Português" e, na consulta por palavra, foi selecionada a opção "Descritor Exato", evitando que fossem levantados estudos com apenas um dos termos ou palavras que compusessem o descritor. Na consulta por índice, elegeu-se o índice permutado, pois esse possibilita saber se a palavra existe no DeCS e visualizar globalmente todos os descritores que a possuem, independente da posição da mesma no termo (Pellizzon, 2004).

Os termos registrados na lista inicial e encontrados como descritor exato no DeCS foram: "comportamento", "terapia comportamental", "medicina do comportamento", "psicologia médica" e "saúde". Os termos "medicina comportamental", "análise do comportamento",

Essa relação de analistas do comportamento de renome na subárea investigada foi obtida por meio da inclusão da combinação "análise do comportamento" AND saúde no campo de busca avançada por assunto no Portal de Periódicos da CAPES e pelo refinamento dos resultados por autor, com consulta ao Currículo Lattes (http:// www.cnpq/br). 
"psicologia da saúde", "análise aplicada do comportamento" e "análise funcional" não foram encontrados como descritores exatos nesse vocabulário controlado. Em função da abrangência dos termos "comportamento" e "terapia comportamental", esses foram descartados. O descritor "saúde" foi mantido para refinar as buscas em associação a outras palavras. Os três descritores restantes foram inseridos na base de dados por meio de diferentes combinações formuladas a partir da hipótese de associação entre variáveis (Volpato, 2007), com a utilização dos operadores booleanos $A N D$ e $O R$. A palavra-chave "análise do comportamento" foi acrescentada aos demais descritores, uma vez que se refere à abordagem que deveria constar nos trabalhos recuperados, ainda que não tenha sido localizada no DeCS. Para delimitar o procedimento, foi verificada a quantidade de trabalhos recuperados em cada combinação e a pertinência dos resultados obtidos em relação ao tema dessa revisão.

Coleta de Dados. Os procedimentos de coleta de dados foram realizados no período de 01/07/15 a 05/07/15. O ano de publicação foi acrescentado por meio da inclusão do $A N D$ e de cada ano do período de 2005 a 2014, independentemente das combinações utilizadas em cada coleta de dados.

Coleta de dados 1. Elegeu-se a combinação "análise do comportamento" $A N D$ "saúde" $O R$ "medicina do comportamento" $O R$ "psicologia médica" como adequada para esse estudo, uma vez que essa tenderia, hipoteticamente, a restringir os resultados para a subárea de interesse, evitando a recuperação da produção analítico-comportamental de maneira demasiadamen- te ampliada e alheia ao objetivo proposto. Tal procedimento fora nomeado como Coleta 1 e os termos da combinação deveriam constar como "palavras" ou "em todos os índices" nos trabalhos.

Coleta de dados 2. Após a constatação do material recuperado, observou-se que os resultados não incluíam publicações de analistas do comportamento de renome na subárea já mencionados. Portanto, esse levantamento estaria incompleto. Delimitou-se um novo procedimento de busca com a manutenção da técnica de associação entre variáveis, mas incluindo termos referentes às especialidades médicas, acrescidos da palavra-chave "psicologia", sendo que os termos que compunham essas combinações deveriam constar no "resumo". O termo "análise do comportamento" foi retirado da estratégia em função dos resultados nulos obtidos na Coleta 1.

A identificação das especialidades médicas foi possível por meio da consulta à Resolução do Conselho Federal de Medicina n. 2.005/2012, que refere 53 especialidades médicas (2012). A inserção dessas especialidades no procedimento em questão foi realizada a fim de localizar os trabalhos de analistas do comportamento que produzem conhecimento em interface com a Medicina. As 53 especialidades foram agregadas em 45 áreas e os termos combinados à palavra-chave "psicologia" corresponderam ao nome da área e/ou a outra palavra relativa ao seu objeto de estudo, com o acréscimo do operador booleano $A N D$. Todas as combinações que compuseram o procedimento da Coleta 2 estão descritas na Figura 1. 


\begin{tabular}{|c|c|c|c|}
\hline \multirow{2}{*}{$\begin{array}{c}\text { Especialidade Médica } \\
\text { Acupuntura }\end{array}$} & \multicolumn{3}{|c|}{ Estratégia de Busca } \\
\hline & Psicologia & $A N D$ & Acupuntura \\
\hline \multirow[t]{2}{*}{ Alergia e Imunologia } & Psicologia & $A N D$ & Alergia \\
\hline & Psicologia & $A N D$ & Imunologia \\
\hline Anestesiologia & $\begin{array}{l}\text { Psicologia } \\
\text { Psicologia }\end{array}$ & $\begin{array}{l}A N D \\
A N D\end{array}$ & $\begin{array}{l}\text { Anestesiologia } \\
\text { Anestesia }\end{array}$ \\
\hline Angiologia & $\begin{array}{l}\text { Psicologia } \\
\text { Psicologia }\end{array}$ & $\begin{array}{l}A N D \\
A N D\end{array}$ & $\begin{array}{l}\text { Angiologia } \\
\text { Vascular }\end{array}$ \\
\hline Cancerologia & $\begin{array}{l}\text { Psicologia } \\
\text { Psicologia }\end{array}$ & $\begin{array}{l}A N D \\
A N D\end{array}$ & $\begin{array}{l}\text { Cancerologia } \\
\text { Câncer }\end{array}$ \\
\hline Cardiologia & $\begin{array}{l}\text { Psicologia } \\
\text { Psicologia }\end{array}$ & $\begin{array}{l}A N D \\
A N D\end{array}$ & $\begin{array}{l}\text { Cardiologia } \\
\text { Coração }\end{array}$ \\
\hline Cirurgia & Psicologia & $A N D$ & Cirurgia \\
\hline Clínica Médica & Psicologia & $A N D$ & Clínica $A N D$ Médica \\
\hline Coloproctologia & Psicologia & $A N D$ & Coloproctologia \\
\hline Dermatologia & Psicologia & $A N D$ & Dermatologia \\
\hline Endocrinologia e Metabologia & $\begin{array}{l}\text { Psicologia } \\
\text { Psicologia }\end{array}$ & $\begin{array}{l}A N D \\
A N D\end{array}$ & $\begin{array}{l}\text { Endocrinologia } \\
\text { Metabologia }\end{array}$ \\
\hline Endoscopia & Psicologia & $A N D$ & Endoscopia \\
\hline Gastroenterologia & Psicologia & $A N D$ & Gastroenterologia \\
\hline Genética Médica & $\begin{array}{l}\text { Psicologia } \\
\text { Psicologia }\end{array}$ & $\begin{array}{l}A N D \\
A N D\end{array}$ & $\begin{array}{c}\text { Genética } A N D \text { Médica } \\
\text { Genética }\end{array}$ \\
\hline Geriatria & Psicologia & $A N D$ & Geriatria \\
\hline Ginecologia e Obstetrícia & $\begin{array}{l}\text { Psicologia } \\
\text { Psicologia }\end{array}$ & $\begin{array}{l}A N D \\
A N D\end{array}$ & $\begin{array}{l}\text { Ginecologia } \\
\text { Obstetrícia }\end{array}$ \\
\hline Hematologia e Hemoterapia & $\begin{array}{l}\text { Psicologia } \\
\text { Psicologia }\end{array}$ & $\begin{array}{l}A N D \\
A N D\end{array}$ & $\begin{array}{l}\text { Hematologia } \\
\text { Hemoterapia }\end{array}$ \\
\hline Homeopatia & Psicologia & $A N D$ & Homeopatia \\
\hline Infectologia & Psicologia & $A N D$ & Infectologia \\
\hline Mastologia & Psicologia & $A N D$ & Mastologia \\
\hline Medicina de Família e Comunidade & $\begin{array}{l}\text { Psicologia } \\
\text { Psicologia }\end{array}$ & $\begin{array}{l}A N D \\
A N D\end{array}$ & $\begin{array}{c}\text { Medicina } A N D \text { Família } \\
\text { Medicina } A N D \text { Comunidade }\end{array}$ \\
\hline Medicina do Trabalho & Psicologia & $A N D$ & Medicina $A N D$ Trabalho \\
\hline Medicina de Tráfego & Psicologia & $A N D$ & Medicina $A N D$ Tráfego \\
\hline Medicina Esportiva & Psicologia & $A N D$ & Medicina $A N D$ Esportiva \\
\hline Medicina Física e Reabilitação & $\begin{array}{l}\text { Psicologia } \\
\text { Psicologia }\end{array}$ & $\begin{array}{l}A N D \\
A N D\end{array}$ & $\begin{array}{c}\text { Medicina } A N D \text { Física } \\
\text { Medicina } A N D \text { Reabilitação }\end{array}$ \\
\hline Medicina Intensiva & Psicologia & $A N D$ & Medicina $A N D$ Intensiva \\
\hline Medicina Legal e Perícia Médica & $\begin{array}{l}\text { Psicologia } \\
\text { Psicologia }\end{array}$ & $\begin{array}{l}A N D \\
A N D\end{array}$ & $\begin{array}{l}\text { Medicina } A N D \text { Legal } \\
\text { Perícia } A N D \text { Médica }\end{array}$ \\
\hline Medicina Nuclear & Psicologia & $A N D$ & Medicina $A N D$ Nuclear \\
\hline
\end{tabular}




\begin{tabular}{|c|c|c|c|}
\hline \multirow{2}{*}{$\begin{array}{c}\text { Especialidade Médica } \\
\text { Medicina Preventiva e Social }\end{array}$} & \multicolumn{3}{|c|}{ Estratégia de Busca } \\
\hline & $\begin{array}{l}\text { Psicologia } \\
\text { Psicologia }\end{array}$ & $\begin{array}{l}A N D \\
A N D\end{array}$ & $\begin{array}{l}\text { Medicina } A N D \text { Preventiva } \\
\text { Medicina } A N D \text { Social }\end{array}$ \\
\hline Nefrologia & Psicologia & $A N D$ & Nefrologia \\
\hline Neurocirurgia & Psicologia & $A N D$ & Neurocirurgia \\
\hline Neurologia & Psicologia & $A N D$ & Neurologia \\
\hline Nutrologia & Psicologia & $A N D$ & Nutrologia \\
\hline Oftalmologia & Psicologia & $A N D$ & Oftalmologia \\
\hline Ortopedia e Traumatologia & $\begin{array}{l}\text { Psicologia } \\
\text { Psicologia }\end{array}$ & $\begin{array}{l}A N D \\
A N D\end{array}$ & $\begin{array}{c}\text { Ortopedia } \\
\text { Traumatologia }\end{array}$ \\
\hline Otorrinolaringologia & Psicologia & $A N D$ & Otorrinolaringologia \\
\hline Patologia & Psicologia & $A N D$ & Patologia \\
\hline $\begin{array}{c}\text { Patologia Clínica e Medicina } \\
\text { Laboratorial }\end{array}$ & $\begin{array}{l}\text { Psicologia } \\
\text { Psicologia }\end{array}$ & $\begin{array}{l}A N D \\
A N D\end{array}$ & $\begin{array}{c}\text { Patologia } A N D \text { Clínica } \\
\text { Medicina } A N D \text { Laboratorial }\end{array}$ \\
\hline Pediatria & Psicologia & $A N D$ & Pediatria \\
\hline Pneumologia & Psicologia & $A N D$ & Pneumologia \\
\hline Psiquiatria & Psicologia & $A N D$ & Psiquiatria \\
\hline $\begin{array}{c}\text { Radiologia e Diagnóstico por } \\
\text { Imagem }\end{array}$ & $\begin{array}{l}\text { Psicologia } \\
\text { Psicologia }\end{array}$ & $\begin{array}{l}A N D \\
A N D\end{array}$ & $\begin{array}{c}\text { Radiologia } \\
\text { Diagnóstico } A N D \text { Imagem }\end{array}$ \\
\hline Radioterapia & Psicologia & $A N D$ & Radioterapia \\
\hline Reumatologia & Psicologia & $A N D$ & Reumatologia \\
\hline Urologia & Psicologia & $A N D$ & Urologia \\
\hline
\end{tabular}

Figura 1. Estratégias de Busca da Coleta 2.

Critérios de Inclusão e de Exclusão dos Artigos Encontrados. Os critérios de inclusão consistiram na publicação ter ocorrido no período de 2005 a 2014, no idioma Português, e que remetesse a artigos científicos analítico-comportamentais com enfoque na Psicologia da Saúde. Foram excluídos resumos: (a) repetidos; (b) incompletos (base de dados não fornecia o resumo por completo); (c) de outras áreas (Odontologia, Medicina, Enfermagem, Educação Física, etc.); (d) que não se referiam a trabalhos com enfoque analítico-comportamental (behaviorista radical); (e) que não foram escritos por psicólogos, ainda que abordassem questões referentes à subárea pesquisada; (f) que não correspondiam à atuação da Psicologia; e (g) de autores brasileiros sem afiliação institucional no próprio país. Esse último critério foi possível por meio da consulta ao
Currículo Lattes dos pesquisadores. Os critérios de exclusão foram adaptados de Ferreira, Soares, Orlandini, e Sabião (2008).

Registro de Dados Coletados. Em ambas as coletas, primeiramente, os descritores e palavras-chave foram inseridos no portal da BVS-Psi Brasil. Posteriormente, os resultados eram registrados em uma planilha do Microsoft Excel e os resumos que constavam no Index Psi Periódicos Técnico-Científicos, SciELO e LILACS foram consultados para leitura na íntegra. As listas com esses resumos foram impressas, aumentando o controle na seleção dos estudos a serem analisados. Nessa etapa, a consulta dos artigos completos foi feita nos casos em que não foi possível identificar os critérios de inclusão e de exclusão da publicação em estudo exclusivamente pelo resumo. 
Definida a pertinência do material, o download do artigo científico completo foi realizado, bem como a leitura desse na íntegra, com a posterior inserção dos dados coletados em uma planilha desenvolvida no Microsoft Excel a partir das seis variáveis de estudo: Ano de Publicação, Tipo de Estudo, Objetivos, Participantes, Procedimentos e Conclusões.

Análise dos Dados. Para a análise quantitativa, foi utilizado o software SPSS (Wagner, 2004) diante dos resultados das variáveis "Ano de Publicação" e "Tipo de Estudo". As variáveis "Objetivos", "Participantes", "Procedimentos" e "Conclusões" foram analisadas qualitativamente, a partir da construção de uma figura para cada publicação, sumarizando os dados coletados.

\section{Resultados}

Foram lidos, ao todo, 789 resumos a partir dos dois procedimentos empregados nas três bases de dados. Na Coleta 1 , seis artigos analítico- -comportamentais foram recuperados e, na Coleta 2, apenas um artigo científico da interface de interesse foi selecionado, pertencente à área de Psiquiatria, totalizando sete publicações, o que representa $0,9 \%$ do total de arquivos encontrados. Desses estudos, $75 \%$ correspondiam a pesquisas aplicadas, $12,5 \%$ a estudos experimentais e $12,5 \%$ a estudos conceituais, o que denota a predominância da literatura da Análise do Comportamento Aplicada referente a questões relacionadas à área médica.

De acordo com a distribuição anual da produção científica da subárea na década estudada, verificou-se que a publicação ocorreu em frequência similar, sendo de $12,5 \%$ ao ano, com variação para 25\% em 2010 e 2011, dentre o total de artigos recuperados. A base de dados on-line na qual fora recuperada cerca da metade dos artigos científicos analisados referiu-se ao Index Psi Periódicos Técnico-Científicos, embora 59,8\% dos resumos encontrados tenham sido obtidos por meio do LILACS. A Figura 2 sumariza informações gerais dos sete artigos.

\begin{tabular}{lccc}
\hline Autor(es) & Periódico & ISSN & Ano de Publicação \\
\hline Ferreira, Mendonça e Lobão & Estudos de Psicologia & $1982-0275$ & 2007 \\
Witter $^{\mathrm{a}}$ & Estudos de Psicologia & $1982-0275$ & 2008 \\
Wechsler e Amaral & Psicologia em Estudo & $1807-0329$ & 2010 \\
Vasconcellos, Rocha e Maciel & ConScientiae Saúde & $1677-1028$ & 2010 \\
Silva e Zakir & Estudos de Psicologia & $1982-0275$ & 2011 \\
Ceppi e Benvenuti & Revista de Psiquiatria Clínica & $0101-6083$ & 2011 \\
Brum e Carrara & Estudos de Psicologia & $1982-0275$ & 2012 \\
\hline
\end{tabular}

Nota. ${ }^{\mathrm{a} O}$ currículo dessa autora é composto por trabalhos que abordam diferentes conceitos da Análise do Comportamento. Contudo, termos tradicionalmente incompatíveis com uma visão analítico-comportamental foram usados no trabalho. Tal artigo foi mantido na análise em função da autora apresentar diversas publicações na área e se reportar à abordagem analítico-comportamental.

Figura 2. Artigos Analítico-Comportamentais Recuperados.

A análise dos objetivos a que os trabalhos de analistas do comportamento na subárea de Psicologia da Saúde fazem referência permitiu averiguar a permanência do enfoque científico pela busca de relações de controle entre variáveis como principal característica dessas produções. O método descritivo também é predominante, bem como o uso de modelos experimentais, a fim de auxiliar na identificação de relações funcionais e na compreensão de questões aplicadas. Isso ocorre em função dessa abordagem interpretar a descrição e a explicação de fenômenos comportamentais como coincidentes (Skinner, 1961). 
Sobre os procedimentos adotados, esses envolveram: aplicação de testes estatísticos, registros de comportamentos, principalmente para automonitoração, treinos para instalação e fortalecimento de repertórios, bem como treino em análise de contingências e análise funcional. Utilizou-se, ainda, a aplicação de outros procedimentos, i. e., relaxamento, emprego de inventários e questionários para coleta de dados e avaliação de procedimentos (pré e pós-intervenção), observação de comportamentos com base em formulários, organização de grupo-controle e grupo experimental para possibilitar comparações entre variáveis, análise de propostas de tratamento e de instrumentos empíricos de análise funcional, pesquisas eletrônicas e revisões teóricas, com a interpretação feita a partir do referencial skinneriano.

Outro aspecto relevante a ser mencionado refere-se à variedade de populações e protocolos de atendimento descritos nos trabalhos da subárea. Essa característica da produção científica corrobora a descrição do campo de atuação do psicólogo da saúde feita por Miyazaki e Amaral (1995), o qual abrange a atenção a demandas diversas que estejam relacionadas ao continuum saúde/doença, atendidas nos diferentes níveis desse sistema. Kubo e Botomé (2001) complementam a questão ao afirmar que a condição de saúde não é um fenômeno estático, mas um processo complexo e dinâmico, que demanda do psicólogo o conhecimento sobre a rede de inter-relações que o determinam, probabilisticamente. Diante dessas contingências de trabalho, o uso de diferentes instrumentos faz-se crucial e necessário.

A partir das conclusões mencionadas nos estudos em questão, pode-se notar que esses têm considerado a pertinente aplicabilidade e utilidade da Análise do Comportamento à área da saúde, a qual traz um enfoque sistêmico para o entendimento do comportamento humano por meio da inserção da análise funcional nesses contextos. Ainda assim, tais contribuições devem ser mais difundidas e utilizadas, conforme Ferreira et al. (2007).

\section{Discussão}

Com base nos resultados, verificou-se o número restrito de artigos da interface recuperados por meio da BVS-Psi Brasil a partir dos dois procedimentos elaborados. Observou-se que não houve consenso na nomenclatura da subárea da Psicologia da Saúde e que os descritores que a definem, tais como "psicologia da saúde" ou "health psychology", não estão incluídos no vocabulário controlado DeCS. Se existem indefinições conceituais, condições precorrentes ao processo de indexação já podem estar sendo desconsideradas, com consequências à qualidade do controle de publicações proposto pela BVS. Portanto, os pesquisadores dessa subárea devem buscar alternativas para o desenvolvimento de pesquisas mais precisas e advindas de bases consensuais mais válidas (Kerbauy, 2002; Witter, 2008).

As palavras-chave utilizadas pelos pesquisadores da Psicologia da Saúde não estão relacionadas à terminologia da Análise do Comportamento ou a termos genéricos que nomeiam essa subárea dentro da Psicologia, como os descritores utilizados na Coleta 1 . Ao mesmo tempo, as palavras-chave relacionadas às especialidades da Medicina recuperaram trabalhos de cunho eminentemente biológico, tendo como resultado apenas um artigo analítico-comportamental. Consequentemente, os descritores exatos empregados recuperaram apenas parte da literatura científica nacional produzida com o viés de interesse dessa pesquisa.

Por outro lado, o uso de termos técnicos analítico-comportamentais genéricos recupera uma quantidade demasiadamente grande de trabalhos, mas que não estão relacionados, necessariamente, à subárea da Psicologia da Saúde. Os termos "análise funcional" e "terapia comportamental", também utilizados em estudos relacionados à Psicologia da Saúde, e.g., são abrangentes e demandariam o estabelecimento de critérios precisos para a definição de características de pesquisas pertencentes à área clínica e à área da saúde. 
A própria indexação feita a partir de termos genéricos da área da saúde dificulta a busca de literatura aos profissionais e estudantes interessados nas diferentes abordagens psicológicas e, no caso, na Análise do Comportamento. Trabalhos com enfoque psicanalítico, e.g., são recuperados por meio de descritores comuns a estudos analítico-comportamentais. Embora se saiba que essas especificidades sejam de conhecimento de estudiosos da Psicologia, uma base de dados que fosse pautada nessas particularidades contribuiria para a sistematização de conceitos, nomenclaturas, classificações e áreas de atuação. Se o processo de indexação de uma base de dados vinculada à OMS não diferencia esses aspectos, seria interessante rever a adequação do planejamento desse controle de publicações.

Devido à preocupação de analistas do comportamento em entender as contingências que produziram determinada condição de saúde, as intervenções são descritas de maneira a identificar classes de estímulos antecedentes, classes de respostas e classes de estímulos consequentes (ou consequências) de padrões comportamentais relevantes para a ocorrência de eventuais enfermidades. A análise funcional se caracteriza, portanto, como instrumento para a compreensão de eventos comportamentais, baseada na premissa de que variáveis ambientais (variáveis independentes) afetam o comportamento (variável dependente), a depender da interação do organismo com o seu ambiente histórico e com o contexto atual. Segundo Matos (1999), o behaviorista radical trabalha com o comportar-se dentro de contextos, aspecto identificado em todos os artigos.

O analista do comportamento, portanto, é responsável por programar contingências por meio das quais serão obtidos produtos agregados e consequências individuais para toda uma comunidade verbal, o que denota o envolvimento de comportamentos sociais complexos e metacontingências relacionadas às políticas públicas na área da saúde (Brum \& Carrara, 2012). Por isso, o enfoque dado, nos artigos analisados, para a operacionalização de comportamentos e a proposta de inserção de intervenções a partir de programas em saúde.
Outro aspecto comum abordado nos estudos diz respeito à relação entre coerção ou aversividade e alterações nas condições de saúde dos indivíduos. Procedimentos aversivos são tidos como situações estressoras, as quais eliciam respondentes e evocam operantes que podem comprometer a adesão aos tratamentos e favorecer a instalação e/ou fortalecimento de repertórios de fuga-esquiva. Em função desse padrão inviabilizar o contato com reforçadores positivos vinculados à saúde, o psicólogo deve atuar na emissão de instruções adequadas às diferentes condições vivenciadas e na modelagem de repertórios de enfrentamento a serem emitidos pelos pacientes diante de contingências aversivas (Wechsler \& Amaral, 2010).

Silva e Zakir (2011) apontam que pesquisas na área da saúde devem ter maior controle do registro de operantes, com maior número de participantes e fazer uso de diferentes instrumentos de coleta de dados, de maneira a favorecer generalizações. Esse mesmo critério, porém com enfoque terapêutico em garantir a manutenção e a generalização do tratamento em ambientes naturais, é citado nos artigos analítico-comportamentais como imprescindível para a análise da efetividade da intervenção, o que demonstra a importância da avaliação dos resultados, critério definido por Kerbauy (2002) como primordial na formação profissional.

O entrelaçamento de conceitos advindos de diferentes áreas do conhecimento foi outra característica da subárea constatada durante a realização desse estudo, o que exige do psicólogo e/ou pesquisador da área da saúde um desempenho diferenciado para propor intervenções e pesquisas a partir de linguagens e metodologias compatíveis à área médica (Costa, 2010). Para tanto, a formação em cursos de graduação deveria sofrer alterações com o intuito de possibilitar a construção de planos de ação de modo interdisciplinar (Witter, 2008).

Questionamentos a respeito de aspectos técnicos mais específicos, como aqueles referentes aos critérios de análise para a classificação das publicações de acordo com as subáreas da Psicologia, bem como da terminologia utilizada por analistas do comportamento para compor 
o conjunto de palavras-chave de seus artigos científicos, também são pertinentes de serem salientados a partir dessa revisão. Hipotetizou-se que os pesquisadores da área da saúde produzem conhecimento, mas, provavelmente, nem sempre se preocupam com o acesso da comunidade a essa produção, hipótese levantada pela discrepância entre os termos definidos como palavras-chave nos trabalhos encontrados e os termos do DeCS utilizados na indexação.

Analisando os resultados desse estudo, entende-se que a comunidade acadêmico-científica deveria facilitar o acesso dos profissionais e estudantes à informação técnico-científica, o que pode ser feito de duas formas. A primeira é por meio do aumento do número de publicações indexadas em bases de dados de acesso livre e em língua portuguesa. No meio acadêmico, publicações em revistas de acesso restrito, e também nas internacionais, são largamente apoiadas, visto que as mesmas costumam contar com uma avaliação que lhes confere maior credibilidade e, consequentemente, maior valorização do currículo dos pesquisadores. Entretanto, o alcance do objetivo de contribuir para a atuação dos profissionais da área pode ficar comprometido, pois a maior parte dos psicólogos não tem acesso a esse conhecimento.

Outro meio de facilitar o acesso ao conhecimento produzido, mesmo nas bases de dados de acesso livre, é com a aproximação dos critérios de análise desses materiais pelos profissionais da Ciência da Informação e da Análise do Comportamento, a partir da revisão dos descritores exatos utilizados nos vocabulários controlados. Os profissionais e estudantes de Psicologia devem ter noção desses vocabulários, os quais orientam o processo de indexação e, portanto, de recuperação da produção científica, com conceitos e sinônimos referentes a um descritor.

Questiona-se: a Análise do Comportamento deve adaptar sua terminologia e linguagem específicas para os contextos médicos? Seria mais coerente e viável uma adaptação da terminologia dessa abordagem da Psicologia à área da saúde ou o contrário? Quais seriam as contribuições da aproximação sugerida entre a Medicina, a Ciência da Informação e a Análise do Comportamen- to? Que estratégias possibilitariam que a Análise do Comportamento aumentasse a visibilidade das suas produções em bases de dados on-line de acesso livre e, consequentemente, favorecessem "intervenções sistematizadas", de acordo com seu enfoque?

Esse artigo denota a complexidade da elaboração de trabalhos de revisão sistemática, a necessidade da divulgação do conhecimento científico para a comunidade acadêmico-profissional como um todo, a execução de estudos sobre os processos de indexação das publicações científicas nas bases de dados on-line e da importância dos descritores de vocabulários controlados para a devida recuperação. As bases de dados podem auxiliar na sistematização de uma área de conhecimento, a depender de como estão organizadas.

\section{Considerações Finais}

Apesar da inconsistência terminológica da Psicologia da Saúde, dos entraves pertencentes à própria estruturação da BVS-Psi Brasil e das limitações dessa investigação, os resultados sugeriram a pertinente aplicabilidade da Análise do Comportamento à área da saúde em função, sobretudo, das pesquisas nessa abordagem psicológica serem desenvolvidas a partir de variáveis de controle e passíveis de replicação (Rolim, Rocha, \& Moraes, 2009). Entende-se, portanto, que esse sistema psicológico pode contribuir para que as produções nacionais apresentem um enfoque diferenciado e mais coerente com as demandas dessa área. Por consequência, incentiva-se a que analistas do comportamento traduzam a literatura da área da saúde para os referenciais teóricos, experimentais e aplicados da sua abordagem.

Ressalta-se, ainda, que, a partir da presente revisão, foi recuperado um número restrito de pesquisas de analistas do comportamento que trabalham com temas relacionados à área da saúde, entretanto, esses profissionais podem estar publicando em bases de dados de acesso restrito ou, até mesmo, em bases de dados de acesso livre, mas tais pesquisas devem ser recuperadas a partir de estratégias de busca diferentes das que foram utilizadas nessa investigação. Não é pos- 
sível avaliar sistematicamente toda a produção científica da Análise do Comportamento na área da saúde com base nesse estudo, ainda assim, a metodologia adotada, englobando bases de acesso livre, foi válida para indicar uma necessidade que pode interferir diretamente na atuação dos analistas do comportamento na área da saúde: a necessidade do conhecimento ao alcance de todos.

Notou-se que a construção de um efetivo controle da produção científica nacional em Psicologia pela BVS-Psi Brasil exigirá exaustivas pesquisas sobre os processos de indexação nessa base, com a devida revisão dos critérios para o agrupamento dos estudos em função do vocabulário DeCS. Sugere-se, para tanto, o desenvolvimento do trabalho colaborativo e interdisciplinar de profissionais da Ciência da Informação, da Medicina e da Psicologia. Pesquisas de campo que averiguem a possível correlação entre as fontes de informação consultadas e a eficácia das intervenções na Psicologia da Saúde, além de estudos que analisem a qualidade da produção científica divulgada em bases de dados de acesso livre, também podem contribuir à temática e ceder condições às intervenções sistematizadas.

\section{Referências}

Brum, M. M., \& Carrara, K. (2012). História individual e práticas culturais: Efeitos no uso de preservativos por adolescentes. Estudos de Psicologia (Campinas), 29(1), 689-697. doi:10.1590/ S0103-166X2012000500005

Castro, E. K. de., \& Bornholdt, E. (2004). Psicologia da Saúde x Psicologia Hospitalar: Definições e possibilidades de inserção profissional. Psicologia: Ciência e Profissão, 24(3), 48-57. doi:10.1590/S1414-98932004000300007

Ceppi, B., \& Benvenuti, M. (2011). Análise funcional do comportamento autolesivo. Revista de Psiquiatria Clínica, 38(6), 247-253. doi:10.1590/ S0101-60832011000600006

Conselho Federal de Medicina. (2012, 21 dez.). Resolução CFM n ${ }^{\circ}$. 2.005, de 21 de dezembro de 2012. Dispõe sobre a nova redação dos Anexos II e III da Resolução n. 1.973 de 2011. Diário Oficial da União, Seção 1, 937-940. Brasília, DF: Autor. Recuperado em http://www.portalmedico.org.br/resolucoes/CFM/2012/2005_2012.pdf
Conselho Federal de Psicologia. (2006). A História da Psicologia Hospitalar [Número especial]. Psicologia: Ciência e Profissão - Diálogos. Saúde e Psicologia: Os desafios teóricos e práticos e as conquistas no cuidado com o sujeito, 3(4), 20-25. Recuperado em http://site.cfp.org.br/wpcontent/uploads/2007/02/dialogos_4.pdf

Conselho Regional de Psicologia $8^{\mathrm{a}}$ Região. (2010). Levantamento do perfil profissional e das condições de trabalho dos psicólogos do Paraná - CRP-08. Recuperado em http://www.portal. crppr.org.br/download/265.pdf

Costa, A. B., \& Zoltowski, A. P. C. (2014). Como escrever um artigo de revisão sistemática. In $\mathrm{S}$. H. Koller, M. C. P. de P. Couto, \& J. V. Hohendorff (Eds.), Manual de produção cientifica (pp. 55-70). Porto Alegre, RS: Penso.

Costa, Á. L., Jr. (1997). Psicologia da Saúde: Uma proposta de renovação de modelos clínicos tradicionais. Revista Insight-Psicoterapia, 7(80), 24-27.

Costa, Á. L., Jr. (2010, maio). Psicologia da Saúde: Pesquisa e intervenção profissional. Trabalho apresentado no I Congresso de Psicologia e Análise do Comportamento, Londrina, PR, Brasil. Recuperado em http:/www.uel.br/ eventos/cpac/pages/edicoes-anteriores/i-cpac. php

Costa, Á. L., Jr., Doca, F. N. P., Araújo, I., Martins, L., Mundim, L., Penatti, T., \& Sidrim, A. C. (2012). Preparação psicológica de pacientes submetidos a procedimentos cirúrgicos. Estudos de Psicologia (Campinas), 29(2), 271-284. doi:10.1590/S0103-166X2012000200013

Ferreira, E. A. P., Mendonça, M. B., \& Lobão, A. C. (2007). Adesão ao tratamento da urticária crônica. Estudos de Psicologia (Campinas), 24(4), 539-549. doi:10.1590/S0103$-166 \times 2007000400013$

Ferreira, R. E., Soares, M. R. Z., Orlandini, T. F., \& Sabião, L. S. (2008, nov.). Mulheres com câncer de mama: Análise de produção científica a partir de resumos publicados entre 2000 e 2008. Trabalho apresentado no II Encontro Paranaense de Análise do Comportamento, Curitiba, PR, Brasil. Recuperado em http://www.fappr.pr.gov.br/arquivos/File/ projetos08-2008/14124_Anais.pdf

Galvão, C. M., Sawada, N. O., \& Trevizan, M. A. (2004). Revisão sistemática: Recurso que pro- 
porciona a incorporação das evidências na prática da Enfermagem. Revista Latino-Americana de Enfermagem, 12(3), 549-556. doi:10.1590/ S0104-11692004000300014

Glueckauf, R. (1999). Interdivisional Healthcare Committee: Speaking with one voice on cross-cutting issues in Health Care Psychology. Journal of Clinical Psychology in Medical Settings, 6(2), 171-181. doi:10.1023/A:1026292110347

Gorayeb, R., \& Guerrelhas, F. (2003). Sistematização da prática psicológica em ambientes médicos. Revista Brasileira de Terapia Comportamental e Cognitiva, 5(1), 11-19. Recuperado em http:// pepsic.bvsalud.org/scielo.php?pid $=\mathrm{S} 1517$ $-55452003000100003 \&$ script $=$ sci arttext

Jenkins, C. D. (2007). Construindo uma saúde melhor: Um guia para a mudança de comportamento (A. P. Fajardo, Trad.). São Paulo, SP: Artmed. (Original publicado em 2003)

Johnston, J. M. (1996). Distinguishing between applied research and practice. The Behavior Analyst, 19(1), 35-47. Retrieved from http://www.ncbi.nlm.nih.gov/pmc/articles/ PMC2733595/pdf/behavan00019-0036.pdf

Kaplan, R. M. (1990). Behavior as the central outcome in health care. American Psychologist, 45(11), 1211-1220. doi:10.1037/0003-066X.45.11.1211

Kerbauy, R. R. (2002). Comportamento e saúde: Doenças e desafios. Psicologia USP, 13(1), 11-28. doi:10.1590/S0103-65642002000100002

King, M. C. (2006). Preparing Psychology and psychologists for new health care markets. $\mathrm{Ca}$ nadian Psychology, 47(1), 51-56. doi:10.1037/ h0087044

Kubo, O. M., \& Botomé, S. P. (2001). Formação e atuação do psicólogo para o tratamento em saúde e em organizações de atendimento à saúde. Interação em Psicologia, 5, 1-14. doi:10.5380/ psi.v5i1.3319

Luna, S. V. de. (2007). Planejamento de pesquisa: Uma introdução. São Paulo, SP: Editora da Pontifícia Universidade Católica de São Paulo.

Matarazzo, J. D. (1980). Behavioral Health and Behavioral Medicine: Frontiers for a new Health Psychology. American Psychologist, 35(9), $807-$ 817. doi:10.1037/0003-066X.35.9.807

Matarazzo, J. D. (1982). Behavioral Health's challenge to academic, scientific, and professional Psychology. American Psychologist, 37(1), 1-14. doi:10.1037/0003-066X.37.1.1
Matos, M. A. (1999). Com o que o Behaviorismo Radical trabalha. In R. A. Banaco (Ed.), Sobre comportamento e cognição: Questões teóricas, metodológicas e a formação em Análise do Comportamento e Terapia Cognitivista (2a. ed., pp. 45-53). São Paulo, SP: ARBytes.

McNamara, J. R. (1981). Some unresolved challenges facing Psychology's entrance into the health care field. Professional Psychology, 12(3), 391399. doi:10.1037/0735-7028.12.3.391

Ministério da Educação, Coordenação de Aperfeiçoamento de Pessoal de Nível Superior. (2013). Atualiza o WebQualis da Área de Psicologia referente ao ano de 2011 (Comunicado $n$. 001/2013). Brasília, DF: Autor. Recuperado em https://www.capes.gov.br/images/stories/download/avaliacao/Comunicado01-2013webqualispsic.pdf

Miyazaki, M. C. de O. S., \& Amaral, V. L. A. R. (1995). Instituições de saúde. In B. Rangé (Ed.), Psicoterapia comportamental e cognitiva: Pesquisa, prática, aplicações e problemas (pp. 235244). Campinas, SP: Editorial Psy.

Packer, A. L. (2005). A construção coletiva da Biblioteca Virtual em Saúde. Interface - Comunicação, Saúde e Educação, 9(17), 249-272. doi:10.1590/S1414-32832005000200004

Pellizzon, R. de F. (2004). Pesquisa na área da saúde: 1. Base de dados DeCS (Descritores em Ciências da Saúde). Acta Cirúrgica Brasileira, 19(2), 153163. doi:10.1590/S0102-86502004000200013

Pomerleau, O. F. (1979). Behavioral Medicine: The contribution of the Experimental Analysis of Behavior to medical care. American Psychologist, 34(8), 654-663. doi:10.1037/0003$-066 X .34 .8 .654$

Reid, D. H. (1991). Technological behavior analysis and societal impact: A human services perspective. Journal of Applied Behavior Analysis, 24(3), 437-439. doi:10.1901/jaba.1991.24-437

Rolim, G. S., Rocha, R. A. de S., \& Moraes, A. B. A. (2009). O ensino e a pesquisa em Odontopediatria: $\mathrm{O}$ difícil controle de variáveis. $\mathrm{Mu}$ danças - Psicologia da Saúde, 17(1), 17-21. doi:10.15603/2176-1019/mud.v17n1p17-21

Silva, M. E. M., \& Zakir, N. S. (2011). Controle instrucional e relaxamento como preparo psicológico pré-cirúrgico para portadores de cardiopatia. Estudos de Psicologia (Campinas), 28(3), 371379. doi:10.1590/S0103-166X2011000300009 
Skinner, B. F. (1961). The concept of the reflex in the description of behavior. In B. F. Skinner, Cumulative Record [Enlarged edition] (pp. 319-346). New York: Appleton-Century-Crofts.

Smith, T. W., Kendall, P. C., \& Keefe, F. J. (2002). Behavioral Medicine and Clinical Health Psychology: Introduction to the special issue, a view from the decade of behavior. Journal of Consulting and Clinical Psychology, 70(3), 459462. doi:10.1037/0022-006X.70.3.459

Straub, R. O. (2005). Psicologia da Saúde: Uma abordagem biopsicossocial. Porto Alegre, RS: Artmed.

Vasconcellos, M., Rocha, M. C. D. O., \& Maciel, V. H. (2010). Revisão teórica sobre depressão pela Análise do Comportamento e alguns manuais psiquiátricos. ConScientiae Saúde, 9(4), 719725. doi:10.5585/conssaude.v9i4.2145

Volpato, G. L. (2007). Ciência: Da filosofia à publicação. São Paulo, SP: Cultura Acadêmica.
Wagner, M. B. (2004). SPSS passo a passo: Statistical package for the social sciences. Versão 20.0. Caxias do Sul, RS: Editora da Universidade de Caxias do Sul.

Wechsler, A. M., \& Amaral, V. L. A. R. (2010). Dizer $\mathrm{x}$ fazer de mães e filhos em exame médico. Psicologia em Estudo, 15(1), 55-63. doi:10.1590/S1413-73722010000100007

Witter, G. P. (2008). Psicologia da Saúde e produção científica. Estudos de Psicologia (Campinas), 25(4), 577-584. doi:10.1590/S0103166X2008000400012
Recebido: $28 / 01 / 2015$

$1^{a}$ revisão: 09/07/2015

$2^{a}$ revisão: $26 / 08 / 2015$ Aceite final: 27/08/2015 